\title{
Examples of finite free complexes of small rank and small homology
}

by

\author{
SRIKANTH B. IYENGAR \\ University of Utah \\ Salt Lake City, UT, U.S.A.
}

\author{
MARK E. WALKER \\ University of Nebraska \\ Lincoln, NE, U.S.A.
}

\section{Introduction}

In this paper we construct counterexamples to five related conjectures concerning the rank and homology of finite free complexes over commutative noetherian rings, and, in particular, over group algebras of elementary abelian groups.

Conjecture 1.1. Let $k$ be a field of positive characteristic $p$, let $E$ be an elementary abelian $p$-group of rank $r$ and let $k E$ be the corresponding group algebra. If $F$ is a bounded complex of free $k E$-modules of finite rank and $H(F) \neq 0$, then $\operatorname{rank}_{k} H(F) \geqslant 2^{r}$.

Here, $\operatorname{rank}_{k} H(F)$ denotes $\sum_{i} \operatorname{rank}_{k} H_{i}(F)$. Conjecture 1.1 is an algebraic generalization of a conjecture in topology due to Carlsson, recalled in Remark 3.3; see [1, Question 7.3], and [2, §2]. Carlsson proved Conjecture 1.1 when $p=2$ and $r \leqslant 3$; see [16, Theorem 2]. Corollary 3.2 below provides a counterexample whenever $p \geqslant 3$ and $r \geqslant 8$.

The next conjecture concerns a graded polynomial ring $R=k\left[t_{1}, \ldots, t_{d}\right]$ over a field $k$, on indeterminates $t_{1}, \ldots, t_{d}$ of upper degree 2 . A differential graded (DG) $R$-module is a graded $R$-module $F$ equipped with an $R$-linear endomorphism $d$ of $F$ that has (upper) degree 1 and satisfies $d^{2}=0$. Such a DG module is semifree provided there is a chain of graded submodules

$$
0=F(-1) \subseteq F(0) \subseteq F(1) \subseteq \ldots \subseteq \bigcup_{i \geqslant 0} F(i)=F
$$

such that $F(i) / F(i-1)$ is a graded free $R$-module and $d(F(i)) \subseteq F(i-1)$ for all $i$. In particular, ignoring the differential, $F$ itself is a graded free $R$-module; we write $\operatorname{rank}_{R} F$ for its rank. For further details, see, for example, $[8, \S 1.3]$.

Conjecture 1.2. For $R=k\left[t_{1}, \ldots, t_{d}\right]$ as above, if $F$ is a semifree DG $R$-module such that $\operatorname{rank}_{k} H(F)$ is finite and non-zero, then $\operatorname{rank}_{R} F \geqslant 2^{d}$. 
This is a generalization of a topological conjecture due to Halperin; see Remark 4.8. For $d \leqslant 3$ Conjecture 1.2 has been proved by Allday and Puppe [5, Proposition 1.1 and Corollary 1.2]; see also [10, Theorem 5.2 and Remark 5.5]. Walker [27, Theorem 5.3] proved it when char $k \neq 2$ and $H(F)$ is concentrated in even degrees or in odd degrees. Corollary 4.7 below describes counterexamples when char $k \neq 2$ and $d \geqslant 8$; the DG modules constructed have cohomology in degrees 0 and 3 .

A conjecture due to Buchsbaum and Eisenbud [14, Proposition 1.4], and Horrocks [23, Problem 24] predicts that over a local ring $R$ of Krull dimension $d$, any free resolution $F$ of a non-zero module of finite length satisfies $\operatorname{rank}_{R} F_{i} \geqslant\left(\begin{array}{c}d \\ i\end{array}\right)$ for all $i$. In particular, $\operatorname{rank}_{R} F \geqslant 2^{d}$; this last inequality was conjectured by Avramov, see [18, pp. 63], and proved by Walker [26, Theorem 1] when $R$ is a complete intersection whose residual characteristic is not 2 , and also when $R$ is any local ring containing a field of positive characteristic not equal to 2.

Folklore has extended Avramov's conjecture to all finite free complexes.

Conjecture 1.3. If $R$ is a local ring and $F$ is a complex of free $R$-modules with length $_{R} H(F)$ finite and non-zero, then $\operatorname{rank}_{R} F \geqslant 2^{d}$, where $d$ is the Krull dimension of $R$.

For $d \leqslant 3$ this was proved by Avramov, Buchweitz, and Iyengar [10, Theorem 5.2]. Theorem 4.1 below provides counterexamples; the simplest one occurs when $R$ is a regular local ring of dimension 8 and residual characteristic not two.

The next conjecture concerns differential modules. A differential module over a ring $R$ is an $R$-module $F$ equipped with an $R$-linear endomorphism $d$ satisfying $d^{2}=0$. For such an $F$, a free flag consists of a chain of submodules

$$
0=F(-1) \subseteq F(0) \subseteq F(1) \subseteq \ldots \subseteq \bigcup_{i \geqslant 0} F(i)=F
$$

such that $F(i) / F(i-1)$ is a free $R$-module and $d(F(i)) \subseteq F(i-1)$ for all $i$.

Conjecture 1.4. If $R$ is a local ring and $F$ is a differential $R$-module that admits a free flag and has the property that length ${ }_{R} H(F)$ is finite and non-zero, then $\operatorname{rank}_{R} F \geqslant 2^{d}$, where $d$ is the Krull dimension of $R$.

Conjecture 1.4 is stated in [10, Conjecture 5.3] and proven there for $d \leqslant 3$. Given any chain complex of $R$-modules, the direct sum of its components is a differential $R$ module, called its compression. The compression of a free complex admits a free flag. Thus, Conjecture 1.4 implies 1.3, so any counterexample to the latter yields one also to the former; see Corollary 4.6 . 
The final conjecture concerns the sequence of Betti numbers for modules over complete intersection rings. The $i$ th Betti number of a finitely generated module $M$ over a local ring $R$ is the rank of the free $R$-module in degree $i$ in a minimal free resolution of $M$; we denote it by $\beta_{i}^{R}(M)$. A local ring is a complete intersection if its completion is isomorphic to the quotient of a regular local ring by a regular sequence. Over such a ring, the Betti numbers of any finitely generated $R$-module $M$ are eventually given by a quasi-polynomial of period 2; see [21, Corollary 4.2], and also [6, Theorem 4.1] and [9, Theorem 7.3]. In detail, when the projective dimension of $M$ is infinite, there is a positive integer $c$, called the complexity of $M$, a positive real number $\beta$-deg $M$, called the Betti degree of $M$, and polynomials $q_{\mathrm{ev}}$ and $q_{\mathrm{odd}}$ of degree at most $c-2$ such that, for $i \gg 0$, one has

$$
\beta_{i}^{R}(M)=\frac{\beta-\operatorname{deg} M}{2^{c-1}(c-1) !} i^{c-1}+ \begin{cases}q_{\mathrm{ev}}(i), & \text { if } i \text { is even } \\ q_{\mathrm{odd}}(i), & \text { if } i \text { is odd }\end{cases}
$$

In this notation [9, Conjecture 7.5] reads as follows.

Conjecture 1.5. For any finitely generated module $M$ of complexity $c \geqslant 1$ over a complete intersection local ring $R$, one has $\beta$ - $\operatorname{deg} M \geqslant 2^{c-1}$.

This conjecture was motivated by a relationship with Conjecture 1.3 ; see $[9$, Example 7.4]. Avramov and Buchweitz [9, Remark 7.5.1] proved this conjecture when $c \leqslant 2$, and it holds when $q_{\mathrm{ev}}=q_{\mathrm{odd}}$, in particular when $R$ is the localization of a graded ring defined by quadrics [7, Theorem 2.3].

Corollary 4.4 provides counterexamples whenever $R$ has defining relations of order at least 3, Krull dimension zero, embedding dimension at least 8, and residual characteristic not equal to 2 .

The starting point of the construction of our examples is a result on the existence of Lefschetz elements in exterior algebras, recalled in $\S 2$. This connection is present already in the work of Allday and Halperin [3]; see also [4, Example 4.5] by Allday and Puppe, and [19, Corollary 7.2.5] by Félix, Oprea, and Tanré.

\section{Lefschetz elements in exterior algebras}

In this section we recall a basic result concerning exterior algebras that underlies all our constructions. The Hilbert series of a finite-dimensional $\mathbb{Z}$-graded vector space $W=$ $\left\{W_{i}\right\}_{i \in \mathbb{Z}}$ over a field $k$ is the Laurent polynomial

$$
\operatorname{Hilb}_{W}(t):=\sum_{i \in \mathbb{Z}} \operatorname{rank}_{k}\left(W_{i}\right) t^{i}
$$


with non-negative integer coefficients. Evidently $\operatorname{Hilb}_{W}(1)=\operatorname{rank}_{k} W$, and

$$
\operatorname{Hilb}_{\Sigma^{n} W}(t)=t^{n} \operatorname{Hilb}_{W}(t)
$$

where $\Sigma^{n} W$ is the graded $k$-vector space with $\left(\Sigma^{n} W\right)_{i}=W_{i-n}$ for each $i$.

Proposition 2.1. Let $k$ be a field and $\Lambda$ be the exterior algebra of a $k$-vector space with basis $x_{1}, \ldots, x_{n}, y_{1}, \ldots, y_{n}$, in lower degree 1 . Thus $\Lambda_{i}$, the degree $i$ part of $\Lambda$, is the $i$-th exterior power of the given vector space. Set

$$
w:=\sum_{i=1}^{n} x_{i} y_{i} \in \Lambda_{2}
$$

and let $\lambda_{w}: \Lambda \rightarrow \Sigma^{-2} \Lambda$ be the morphism of graded $\Lambda$-modules where $1 \mapsto w$.

If char $k=0$ or char $k>\frac{1}{2}(n+1)$, then the map

$$
\left(\lambda_{w}\right)_{i}: \Lambda_{i} \longrightarrow \Lambda_{i+2}
$$

is injective for $i \leqslant n-1$ and surjective for $i \geqslant n-1$. Moreover, we have

$$
\operatorname{Hilb}_{\operatorname{Coker}\left(\lambda_{w}\right)}(t)=\frac{h(t)}{t^{2}} \quad \text { and } \quad \operatorname{Hilb}_{\operatorname{Ker}\left(\lambda_{w}\right)}(t)=t^{2 n} h\left(\frac{1}{t}\right) \text {, }
$$

where

$$
h(t):=\sum_{i=0}^{n}\left(\left(\begin{array}{c}
2 n \\
i
\end{array}\right)-\left(\begin{array}{c}
2 n \\
i-2
\end{array}\right)\right) t^{i}
$$

and there is an equality

$$
\operatorname{rank}_{k} \operatorname{Coker}\left(\lambda_{w}\right)+\operatorname{rank}_{k} \operatorname{Ker}\left(\lambda_{w}\right)=\left(\begin{array}{c}
2 n+2 \\
n+1
\end{array}\right) .
$$

Proof. See [17, Proposition A.2] for a proof of the assertion concerning the injectivity/surjectivity of multiplication by $w$. Given this, it is elementary to check that the Hilbert series of $\operatorname{Coker}\left(\lambda_{w}\right)$ and $\operatorname{Ker}\left(\lambda_{w}\right)$ are as stated. It remains to note that

$$
\operatorname{rank}_{k} \operatorname{Coker}\left(\lambda_{w}\right)+\operatorname{rank}_{k} \operatorname{Ker}\left(\lambda_{w}\right)=h(1)+h(1)=\left(\begin{array}{c}
2 n+2 \\
n+1
\end{array}\right),
$$

where the second equality can be verified as follows:

$$
\begin{aligned}
2 h(1) & =\sum_{i=0}^{n}\left(\left(\begin{array}{c}
2 n \\
i
\end{array}\right)-\left(\begin{array}{c}
2 n \\
i-2
\end{array}\right)\right)+\sum_{i=n}^{2 n}\left(\left(\begin{array}{c}
2 n \\
i
\end{array}\right)-\left(\begin{array}{c}
2 n \\
i+2
\end{array}\right)\right) \\
& =\left(\begin{array}{c}
2 n \\
n-1
\end{array}\right)+2\left(\begin{array}{c}
2 n \\
n
\end{array}\right)+\left(\begin{array}{c}
2 n \\
n+1
\end{array}\right)=\left(\begin{array}{c}
2 n+1 \\
n
\end{array}\right)+\left(\begin{array}{c}
2 n+1 \\
n+1
\end{array}\right)=\left(\begin{array}{c}
2 n+2 \\
n+1
\end{array}\right) .
\end{aligned}
$$

This completes the proof. 
Remark 2.2. If char $k=0$, then the first assertion in Proposition 2.1 can be proved using the representation theory of $\mathrm{sl}_{2}(k)$, in a manner similar to an argument that appears in the proof of the Hard-Lefschetz theorem found in [20, p. 122].

Indeed, let $x_{1}^{*}, \ldots, x_{n}^{*}, y_{1}^{*}, \ldots, y_{n}^{*}$ be the basis of $\operatorname{Hom}_{k}\left(\Lambda_{1}, k\right)$ dual to the given basis for $\Lambda_{1}$. The elements $x_{i}^{*}$ and $y_{i}^{*}$ induce $k$-linear derivations of degree -1 on $\Lambda$. Set $c:=\sum_{i} y_{i}^{*} x_{i}^{*}$; this is an endomorphism of $\Lambda$ of degree -2 . The restriction of $h:=\left[c, \lambda_{w}\right]$ to $\Lambda_{j}$ is multiplication by $n-j$. We also have $[c, h]=-2 c$ and $\left[\lambda_{w}, h\right]=2 \lambda_{w}$, and thus the operators $\lambda_{w}, c$ and $h$ endow $\Lambda$ with the structure of a $\operatorname{sl}_{2}(k)$-representation such that $\Lambda_{j}$ has weight $n-j$. It follows that $\lambda_{w}^{j}: \Lambda_{n-j} \rightarrow \Lambda_{n+j}$ is an isomorphism for all $j \geqslant 1$; see [25, Chapter IV, Theorem $4(\mathrm{~b})]$.

COROLlary 2.3. Let $k$ be a field with char $k \neq 2$ and $\Lambda$ an exterior algebra on $a$ $k$-vector space of rank $d$. If $d \geqslant 8$, then there is an element $w \in \Lambda_{2}$ such that

$$
\operatorname{rank}_{k} \operatorname{Coker}\left(\lambda_{w}\right)+\operatorname{rank}_{k} \operatorname{Ker}\left(\lambda_{w}\right)=2^{d}-2^{d-6}<2^{d},
$$

where $\lambda_{w}: \Lambda \rightarrow \Sigma^{-2} \Lambda$ is multiplication by $w$.

Proof. Let $X$ be a basis for $\Lambda_{1}$. Select any 8-element subset

$$
X^{\prime}=\left\{x_{1}, \ldots, x_{4}, y_{1}, \ldots, y_{4}\right\}
$$

of $X$, and set $w=\sum_{i} x_{i} y_{i}$. There is an isomorphism of $k$-algebras $\Lambda=\Lambda^{\prime} \otimes_{k} \Lambda^{\prime \prime}$, where $\Lambda^{\prime}$ is the algebra generated by $X^{\prime}$ and $\Lambda^{\prime \prime}$ is the algebra generated by $X \backslash X^{\prime}$. By Proposition 2.1,

$$
\operatorname{rank}_{k} \operatorname{Coker}\left(\lambda_{w}^{\prime}\right)+\operatorname{rank}_{k} \operatorname{Ker}\left(\lambda_{w}^{\prime}\right)=\left(\begin{array}{c}
10 \\
5
\end{array}\right)=252=\left(2^{8}-4\right),
$$

where $\lambda_{w}^{\prime}: \Lambda^{\prime} \rightarrow \Sigma^{-2} \Lambda^{\prime}$ is, as before, multiplication by $w$. There are isomorphisms of $k$-vector spaces

$$
\begin{gathered}
\operatorname{Coker}\left(\lambda_{w}\right) \cong \operatorname{Coker}\left(\lambda_{w}^{\prime}\right) \otimes_{k} \Lambda^{\prime \prime} \\
\operatorname{Ker}\left(\lambda_{w}\right) \cong \operatorname{Ker}\left(\lambda_{w}^{\prime}\right) \otimes_{k} \Lambda^{\prime \prime}
\end{gathered}
$$

from which we deduce that

$$
\operatorname{rank}_{k} \operatorname{Coker}\left(\lambda_{w}\right)+\operatorname{rank}_{k} \operatorname{Ker}\left(\lambda_{w}\right)=2^{d}-2^{d-6} .
$$

Remark 2.4. If char $k=2$, then for every $w \in \Lambda_{\geqslant 1}$ we have $w^{2}=0$, and hence

$$
\operatorname{rank}_{k} \operatorname{Coker}\left(\lambda_{w}\right)+\operatorname{rank}_{k} \operatorname{Ker}\left(\lambda_{w}\right) \geqslant 2^{d} .
$$

Thus, Corollary 2.3 does not extend to char $k=2$; this is why all our examples are in characteristic not equal to 2. 
Remark 2.5. The numbers $\left(\begin{array}{c}2 n+2 \\ n+1\end{array}\right)$, called central binomial coefficients, are related to the Catalan numbers $C_{n}$ by the formula $\left(\begin{array}{c}2 n+2 \\ n+1\end{array}\right)=(n+2) C_{n+1}$. Our counter-examples involve values of $n$ for which the inequality

$$
\left(\begin{array}{c}
2 n+2 \\
n+1
\end{array}\right)<2^{2 n}
$$

holds. As seen in the proof of Corollary 2.3, it holds when $n=4$, and this is the smallest value of $n$ for which it does. It follows from Stirling's formula that

$$
\left(\begin{array}{c}
2 n+2 \\
n+1
\end{array}\right)<2^{2 n} \frac{4}{\sqrt{\pi(n+1)}}
$$

for all $n \geqslant 0$; see [12, equation (1.5)]. In particular, (2.1) holds for all $n \geqslant 5$ too.

Remark 2.6. In $\S 4$ we need versions of Proposition 2.1 and Corollary 2.3 in which the $x_{i}$ 's and $y_{i}$ 's have lower degree -1 . In this case, we switch to upper indexing: by convention, for a graded object $X$, the component of upper degree $i$, written $X^{i}$, is defined to be $X_{-i}$. Note that $\left(\Sigma^{n} X\right)^{i}=X^{n+i}$. We define the Hilbert series of a graded vector space $W=\left\{W^{i}\right\}_{i \in \mathbb{Z}}$ satisfying $W^{i}=0$ for $i \ll 0$ to be $\operatorname{Hilb}_{W}(t)=\sum_{i} \operatorname{rank}_{k}\left(W^{i}\right) t^{i}$.

When $x_{i}, y_{i} \in \Lambda^{1}$ for all $i$, the map in Proposition 2.1 takes the form

$$
\lambda_{w}: \Lambda \longrightarrow \Sigma^{2} \Lambda
$$

The Hilbert series of the cokernel and kernel of this morphism are still the same:

$$
\operatorname{Hilb}_{\operatorname{Coker}\left(\lambda_{w}\right)}(t)=\frac{h(t)}{t^{2}} \quad \text { and } \quad \operatorname{Hilb}_{\operatorname{Ker}\left(\lambda_{w}\right)}(t)=t^{2 n} h\left(\frac{1}{t}\right)
$$

where $h(t)$ is as in Proposition 2.1. The equation in Corollary 2.3 remains valid.

\section{Homology of finite free complexes}

In this section, we construct counterexamples to Conjecture 1.1.

Let $(R, \mathfrak{m}, k)$ be a (commutative, noetherian) local ring $R$, with maximal ideal $\mathfrak{m}$ and residue field $k$. The embedding dimension of $R$ is the integer

$$
\operatorname{emb} \operatorname{dim} R=\operatorname{rank}_{k}\left(\mathfrak{m} / \mathfrak{m}^{2}\right)
$$

and the codimension of $R$ is the integer

$$
\operatorname{codim} R=\operatorname{emb} \operatorname{dim} R-\operatorname{dim} R .
$$


The $\mathfrak{m}$-adic completion of $R$ has the form $Q / I$, where $(Q, \mathfrak{n}, k)$ is a regular local ring and $I \subseteq \mathfrak{n}^{2} ;$ see $[13, \S 2.3]$. For any such presentation, we have

$$
\operatorname{emb} \operatorname{dim} R=\operatorname{dim} Q \quad \text { and } \quad \operatorname{codim} R=\operatorname{dim} Q-\operatorname{dim} R
$$

We say $R$ is a complete intersection if $\operatorname{codim} R=\operatorname{rank}_{k}(I / \mathfrak{n} I)$ or, equivalently, if $I$ can be generated by a $Q$-regular sequence; see [13, Theorem 2.3.3].

In the sequel, given a complex $X$ of $R$-modules with differential $d^{X}$ and an integer $m$, the shifted graded module $\Sigma^{m} X$ is a complex with differential $d^{\Sigma^{m} X}=(-1)^{m} d^{X}$. A finite free complex of $R$-modules is a complex of the form

$$
0 \longrightarrow F_{b} \longrightarrow \ldots \longrightarrow F_{a} \longrightarrow 0
$$

with each $F_{i}$ free of finite rank.

THEOREM 3.1. Let $(R, \mathfrak{m}, k)$ be a complete intersection of codimension $r$. If $r \geqslant 8$ and char $k \neq 2$, then there is a finite free complex of $R$-modules $F$ with

$$
\operatorname{length}_{R} H(F)=2^{r}-2^{r-6} \text {. }
$$

Proof. Let $K$ the Koszul complex on a minimal set of generators of $\mathfrak{m}$. Then, $K$ is a commutative DG $R$-algebra, $H_{1}(K)$ is a $k$-vector space of dimension $r$, and there is an isomorphism of graded $k$-algebras

$$
H(K) \cong \Lambda_{k}\left(H_{1}(K)\right)
$$

see, for instance, [13, Theorem 2.3.1]. Set $\Lambda=H(F)$, let $w \in H_{2}(K)$ be an element as in Corollary 2.3, and let $z \in K_{2}$ be a cycle representing $w$. Since $K$ is a DG algebra, multiplication by $z$ determines a morphism of DG $K$-modules

$$
\begin{gathered}
\lambda_{z}: K \longrightarrow \Sigma^{-2} K, \\
u \longmapsto u z .
\end{gathered}
$$

Set $F:=\operatorname{cone}\left(\lambda_{z}\right)$, the mapping cone of the morphism $\lambda_{z}$. There is an exact sequence of DG $K$-modules

$$
0 \longrightarrow \Sigma^{-2} K \longrightarrow F \longrightarrow \Sigma K \longrightarrow 0
$$

Since $K$ is a finite free $R$-complex so is $F$. The associated exact sequence in homology has the form

$$
\ldots \longrightarrow H_{j}(K) \stackrel{\lambda_{w}}{\longrightarrow} H_{j+2}(K) \longrightarrow H_{j}(F) \longrightarrow H_{j-1}(K) \stackrel{\lambda_{w}}{\longrightarrow} H_{j+1}(K) \longrightarrow \ldots
$$


Thus, there is an exact sequence of graded $R$-modules

$$
0 \longrightarrow \operatorname{Coker}\left(H(K) \stackrel{\lambda_{w}}{\longrightarrow} \Sigma^{-2} H(K)\right) \longrightarrow H(F) \longrightarrow \operatorname{Ker}\left(\Sigma H(K) \stackrel{\lambda_{w}}{\longrightarrow} \Sigma^{-1} H(K)\right) \longrightarrow 0 .
$$

It follows that

$$
\operatorname{length}_{R} H(F)=\operatorname{rank}_{k} \operatorname{Coker}\left(\lambda_{w}\right)+\operatorname{rank}_{k} \operatorname{Ker}\left(\lambda_{w}\right)=2^{r}-2^{r-6},
$$

where the second equality is by the choice of $w$; see Corollary 2.3 .

Corollary 3.2. Let $p$ be an odd prime, $k$ a field of characteristic $p$, and $E$ an elementary abelian $p$-group of rank $r$. If $r \geqslant 8$, there is a finite complex $F$ of free $k E$ modules such that $\operatorname{rank}_{k} H(F)<2^{r}$. Thus, Conjecture 1.1 fails when $p$ is odd.

Proof. There is an isomorphism of $k$-algebras

$$
k E \cong \frac{k\left[t_{1}, \ldots, t_{r}\right]}{\left(t_{1}^{p}, \ldots, t_{r}^{p}\right)}
$$

so that $k E$ is a complete intersection of codimension $r$. The result thus follows from Theorem 3.1, since $\operatorname{rank}_{k} M=\operatorname{length}_{k E} M$ for every $k E$-module $M$.

Remark 3.3. Conjecture 1.1 is extrapolated from a conjecture by Carlsson [16, p. 333] (also [15, Conjecture I.3]) predicting that, if a finite CW complex $X$ admits a free, cellular $E$-action, then the total rank of its singular homology with $\mathbb{Z} / p$-coefficients, $H_{*}(X, \mathbb{Z} / p)$, is at least $2^{r}$. In this situation, $H_{*}(X, \mathbb{Z} / p)$ is realized as the homology of a complex $F$ of $k E$-modules satisfying the hypotheses of Conjecture 1.1 with $k=\mathbb{Z} / p$. Thus, Conjecture 1.1 implies Carlsson's conjecture, but we do not know whether the complex $F$ in Corollary 3.2 arises from a space with a free $E$-action.

\section{Total rank and Betti degree of complexes}

In this section we construct counterexamples to Conjectures 1.2-1.5.

For any local ring $(R, \mathfrak{m}, k)$ one has an inequality

$$
\operatorname{length}_{R}\left(R / \mathfrak{m}^{3}\right) \leqslant\left(\begin{array}{c}
\operatorname{emb} \operatorname{dim} R+1 \\
2
\end{array}\right) \text {. }
$$

When equality holds, we say that the defining relations of $R$ are of order at least 3 . This is equivalent to the condition that, in some presentation of the $\mathfrak{m}$-adic completion of $R$ as $Q / I$, for a regular local ring $(Q, \mathfrak{n}, k)$, one has $I \subseteq \mathfrak{n}^{3}$.

Henceforth, $(R, \mathfrak{m}, k)$ will be a complete intersection; see the start of $\S 3$ for the meaning. Let $M$ be an $R$-complex with the $R$-module $H(M)$ finitely generated. As for 
modules, the Betti numbers $\left\{\beta_{i}^{R}(M)\right\}_{i \in \mathbb{Z}}$ of $M$ are the ranks of the free modules in a minimal free resolution of $M$ (see $[24, \S 1.1])$, and can be computed as

$$
\beta_{i}^{R}(M)=\operatorname{rank}_{k} \operatorname{Ext}_{R}^{i}(M, k)
$$

These numbers are finite for all $i$ and are equal to zero for $i \ll 0$. The Poincaré series of $M$ is the generating series

$$
\mathrm{P}_{M}^{R}(t):=\sum_{i \in \mathbb{Z}} \beta_{i}^{R}(M) t^{i} \in \mathbb{Z}[|t|]\left[t^{-1}\right] .
$$

There exist an integer $0 \leqslant c \leqslant \operatorname{codim} R$ and a Laurent polynomial $p_{M}(t)$ with integer coefficients satisfying $p_{M}(1) \neq 0$ such that

$$
\mathrm{P}_{M}^{R}(t)=\frac{p_{M}(t)}{\left(1-t^{2}\right)^{c}} .
$$

This result is due to Gulliksen [21, Corollary 4.2]; see also [8, Theorem 9.2.1]. The integer $c$ is the complexity of $M$; Remark 4.3 reconciles this definition with the one given in the introduction.

We are interested in the integer $p_{M}(1)$. If $c=0$, then

$$
p_{M}(1)=\mathrm{P}_{M}^{R}(1)=\sum_{i} \beta_{i}^{R}(M),
$$

the total Betti number of $M$. In view of this, when $\operatorname{codim} R=0$, the next result provides counterexamples to Conjecture 1.3.

THEOREM 4.1. Let $(R, \mathfrak{m}, k)$ be a complete intersection whose defining relations have order at least 3. If $\operatorname{char} k \neq 2$ and $e:=\operatorname{emb} \operatorname{dim} R$ is at least 8 , then there exists a complex $F$ with $H_{0}(F) \cong k \cong H_{1}(F)$ and $H_{i}(F)=0$ for all $i \neq 0,1$, with the property that

$$
p_{F}(1)=2^{e}-2^{e-6} \text {. }
$$

Moreover, when codim $R \geqslant 1$, there exists a finitely generated $R$-module $M$ with

$$
p_{M}(1)=2^{e}-2^{e-6}
$$

Proof. Set $c=\operatorname{codim} R$. Since the defining relations of $R$ have order at least 3 , there is an isomorphism of $k$-algebras

$$
\operatorname{Ext}_{R}(k, k) \cong \Lambda \otimes_{k} S,
$$


where $\Lambda$ is an exterior algebra generated by $e$ elements of upper degree 1 , and $S$ is a polynomial algebra generated by $c$ elements of upper degree 2; see [8, Example 10.2.3]. Choose $w \in \Lambda^{2}$ as in Corollary 2.3; see also Remark 2.6. Viewed as an element in $\operatorname{Ext}_{R}(k, k)$, the element $w \otimes 1$ represents a morphism of $R$-complexes

$$
\zeta: \Sigma^{-2} X \longrightarrow X,
$$

where $X$ is a minimal $R$-free resolution of $k$. Let $F=\Sigma \operatorname{cone}(\zeta)$, so there is an exact sequence of $R$-complexes

$$
0 \longrightarrow \Sigma X \longrightarrow F \longrightarrow X \longrightarrow 0
$$

The induced exact sequence in homology

$$
\ldots \longrightarrow H_{i+1}(X) \longrightarrow H_{i-1}(X) \longrightarrow H_{i}(F) \longrightarrow H_{i}(X) \longrightarrow H_{i-2}(X) \longrightarrow \ldots
$$

gives $H_{0}(F) \cong k \cong H_{1}(F)$ and $H_{i}(F)=0$ for all $i \neq 0,1$.

Under the isomorphism (4.2), the endomorphism $\operatorname{Ext}_{R}(\zeta, k)$ of $\operatorname{Ext}_{R}(k, k)$ induced by $\zeta$ corresponds to the map

$$
\lambda_{w} \otimes 1: \Lambda \otimes_{k} S \longrightarrow \Sigma^{2} \Lambda \otimes_{k} S,
$$

and thus there is an exact sequence of $\Lambda \otimes_{k} S$-modules

$$
0 \longrightarrow \Sigma^{-2} \operatorname{Coker}\left(\lambda_{w}\right) \otimes_{k} S \longrightarrow \operatorname{Ext}_{R}(F, k) \longrightarrow \Sigma^{-1} \operatorname{Ker}\left(\lambda_{w}\right) \otimes_{k} S \longrightarrow 0
$$

As a sequence of graded $S$-modules, this sequence splits and yields an isomorphism

$$
\operatorname{Ext}_{R}(F, k) \cong W \otimes_{k} S
$$

of graded $S$-modules, where $W$ is the graded $k$-vector space $\Sigma^{-2} \operatorname{Coker}\left(\lambda_{w}\right) \oplus \Sigma^{-1} \operatorname{Ker}\left(\lambda_{w}\right)$. Since the generating series of $S$ is $1 /\left(1-t^{2}\right)^{c}$, we have

$$
\mathrm{P}_{F}^{R}(t)=\frac{\sum_{i} \operatorname{rank}_{k}\left(W^{i}\right) t^{i}}{\left(1-t^{2}\right)^{c}} .
$$

Evaluated at $t=1$, the numerator equals $\operatorname{rank}_{k} W$, which is non-zero because $W$ is nonzero. This justifies the first equality below; the second one is from Corollary 2.3:

$$
p_{F}(1)=\operatorname{rank}_{k} W=\operatorname{rank}_{k} \operatorname{Coker}\left(\lambda_{w}\right)+\operatorname{rank}_{k} \operatorname{Ker}\left(\lambda_{w}\right)=2^{e-8}\left(2^{8}-4\right) .
$$

This proves the first assertion. 
Assume $c \geqslant 1$, so that $R$ is not regular. From (4.3) it follows that the complexity of $F$ equals $c$ and that

$$
p_{F}(t)=\sum_{i} \operatorname{rank}_{k}\left(W^{i}\right) t^{i}
$$

Let $G$ be a minimal free resolution of $F$ and set $M:=\operatorname{Coker}\left(G_{2} \rightarrow G_{1}\right)$. Since $H_{i}(G)=$ $H_{i}(F)=0$ for $i \geqslant 2$, the complex $\Sigma^{-1}\left(G_{\geqslant 1}\right)$ is a minimal free resolution of $M$, and hence

$$
\mathrm{P}_{M}^{R}(t)=\frac{\mathrm{P}_{F}^{R}(t)-\mathrm{P}_{F}^{R}(0)}{t}=\frac{\mathrm{P}_{F}^{R}(t)-1}{t}=\frac{\left(p_{F}(t)-\left(1-t^{2}\right)^{c}\right) t^{-1}}{\left(1-t^{2}\right)^{c}} .
$$

This implies that the complexity of $M$ is also $c$, and, since $c \geqslant 1$, this yields the first equality below:

$$
p_{M}(1)=p_{F}(1)=\operatorname{rank}_{k} W=2^{e}-2^{e-6} .
$$

The remaining equalities have already been justified.

Remark 4.2. In the course of the proof of the preceding result, we have in fact calculated the Poincaré series of the complex $F$. It is

$$
\mathrm{P}_{F}^{R}(t)=\frac{(1+t)^{e-8}\left(1+8 t+27 t^{2}+48 t^{3}+42 t^{4}+42 t^{5}+48 t^{6}+27 t^{7}+8 t^{8}+t^{9}\right)}{\left(1-t^{2}\right)^{c}} .
$$

Using (4.4), one can also compute the Poincaré series of $M$.

Remark 4.3. Let $R$ be a complete intersection, $M$ an $R$-complex with $H(M)$ finitely generated, and $c$ its complexity. If $c \geqslant 1$, then from (4.1) one gets that there are polynomials $q_{\mathrm{ev}}$ and $q_{\mathrm{odd}}$ of degree at most $c-2$ such that, for $i \gg 0$, one has

$$
\beta_{i}^{R}(M)=\frac{\beta-\operatorname{deg} M}{2^{c-1}(c-1) !} i^{c-1}+ \begin{cases}q_{\mathrm{ev}}(i), & \text { if } i \text { is even } \\ q_{\mathrm{odd}}(i), & \text { if } i \text { is odd }\end{cases}
$$

See also [9, Theorem 7.3]. It follows that the coefficient of $t^{i}$ in $\left(1-t^{2}\right)^{c-1} \mathrm{P}_{M}^{R}(t)$ is $\beta$-deg $M$ for $i \gg 0$; that is to say, there are equalities

$$
\frac{p_{M}(t)}{1-t^{2}}=\left(1-t^{2}\right)^{c-1} \mathrm{P}_{M}^{R}(t)=\frac{\beta-\operatorname{deg} M}{1-t}+l(t)
$$

for some Laurent polynomial $l(t)$. In particular, there is an equality

$$
p_{M}(1)=2 \beta-\operatorname{deg} M
$$

In view of this equality, when $\operatorname{dim} R=0$, that is to say, when $\operatorname{emb} \operatorname{dim} R=\operatorname{codim} R$, Theorem 4.1 specializes to the following statement. 
COROLlary 4.4. Let $R$ be a complete intersection with defining relations of order at least 3 and $\operatorname{dim} R=0$. If $\operatorname{char} k \neq 2$ and $c:=\operatorname{codim} R \geqslant 8$, then there exists a finitely generated $R$-module $M$ with $\beta$-deg $M<2^{c-1}$. Thus, Conjecture 1.5 fails.

Remark 4.5. Let $n$ be a positive integer, $(R, \mathfrak{m}, k)$ be a regular local ring of dimension $2 n$, and assume that char $k>\frac{1}{2}(n+1)$. Then, $\operatorname{Ext}_{R}(k, k)$ is the exterior algebra on a $k$-vector space of rank $2 n$. Let $w$ be as in Proposition 2.1 and $G$ be the complex constructed from $w$ as in the proof of Theorem 4.1 above. A direct computation using Proposition 2.1 yields

$$
\mathrm{P}_{G}^{R}(t)=h(t)+t^{2 n+1} h\left(\frac{1}{t}\right)
$$

Hence, the sequence of Betti numbers of $G$ is palindromic. By construction of $G$, the module $M:=\operatorname{Coker}\left(G_{2} \rightarrow G_{1}\right)$ fits into an extension

$$
0 \longrightarrow k \longrightarrow M \longrightarrow \mathfrak{m} \longrightarrow 0
$$

The projective dimension of $M$ equals $2 n$. The $R$-module $M$ is locally free on the punctured spectrum, and hence the same is true of its syzygy modules, $\Omega_{R}^{d}(M)$. The Poincaré series of $M$ is $\left(\mathrm{P}_{G}^{R}(t)-1\right) / t$ (see (4.4)), so the ranks of its syzygy modules are

$$
\operatorname{rank}_{R} \Omega_{R}^{d}(M)= \begin{cases}\left(\begin{array}{c}
2 n \\
d
\end{array}\right)-\left(\begin{array}{c}
2 n \\
d-1
\end{array}\right), & \text { for } 0 \leqslant d \leqslant n, \\
\left(\begin{array}{c}
2 n \\
d
\end{array}\right)-\left(\begin{array}{c}
2 n \\
d+1
\end{array}\right), & \text { for } n+1 \leqslant d \leqslant 2 n .\end{cases}
$$

The projective dimension of $\Omega_{R}^{d}(M)$ is $2 n-d$ and its depth is $d$. This computation has a bearing on [23, Question 25]. Indeed, fix $1 \leqslant d \leqslant 2 n-1$ and set

$$
s(d):=\min _{L}\left\{\operatorname{rank}_{R} L\right\}
$$

where the minimum is taken over all the $L$ that are finitely generated, of depth $d$, and locally free on the punctured spectrum. For $d=n$, the computation above yields

$$
s(n) \leqslant \operatorname{rank}_{R} \Omega_{R}^{n}(M)=\left(\begin{array}{c}
2 n \\
n
\end{array}\right)-\left(\begin{array}{c}
2 n \\
n-1
\end{array}\right) .
$$

This is much better than the bound $s(n) \leqslant\left(\begin{array}{c}2 n-1 \\ n-1\end{array}\right)$ given by the $n$th syzygy of $k$.

Corollary 4.6. Let $(R, \mathfrak{m}, k)$ be a regular local ring of dimension d. If char $k \neq 2$ and $d \geqslant 8$, then there is a differential $R$-module $D$ such that length ${ }_{R} H(D)$ is non-zero and finite, $D$ admits a free flag and $\operatorname{rank}_{R} D<2^{d}$. Thus, Conjecture 1.4 fails. 
Proof. Let $D$ be the compression (see the introduction) of a minimal resolution $G$ of the complex $F$ constructed in Theorem 4.1. Then, $D$ is a differential $R$-module with homology $H(G) \cong H(F) \cong k^{2}$. In particular, the homology of $D$ is non-zero and of finite length. Moreover, $D$ has a free flag because it is the compression of a free complex; see $[10, \S 2.8(6)]$. The minimality of $G$ gives $\operatorname{rank}_{R} D=\operatorname{rank}_{R}(G)=\sum_{i} \beta_{i}^{R}(F)<2^{d}$.

Corollary 4.7. Let $R:=k\left[t_{1}, \ldots, t_{d}\right]$ be the polynomial ring over a field $k$ in indeterminates $t_{1}, \ldots, t_{d}$ of upper degree 2 , viewed as a DG algebra with zero differential. If char $k \neq 2$ and $d \geqslant 8$, then there is a semifree DG $R$-module $G$ with $\mathrm{H}^{0}(G) \cong k \cong \mathrm{H}^{3}(G)$ and $\mathrm{H}^{j}(G)=0$ for all $j \neq 0,3$ and such that $\operatorname{rank}_{R} G<2^{d}$. Thus, Conjecture 1.2 fails.

Proof. We construct $G$ by mimicking the argument of Theorem 4.1 in the setting of DG-modules. In detail, let $X$ be the Koszul resolution of $k$, given by the commutative DG- $R$-algebra generated by elements $e_{1}, \ldots, e_{d}$ of upper degree 1 and $d\left(e_{i}\right)=t_{i}$. Since $X$ is quasi-isomorphic to $k$ as DG- $R$-modules, $H\left(\operatorname{End}_{R}(X)\right)$ is an exterior algebra on $d$ elements of upper degree -1 . Let $\zeta: \Sigma^{2} X \rightarrow X$ be a degree- $(-2)$ cycle in $\operatorname{End}_{R}(X)$ that represents the degree- $(-2)$ element $w$ of the exterior algebra $H\left(\operatorname{End}_{R}(X)\right)$ given by Corollary 2.3, and define $F=\Sigma^{-3} \operatorname{cone}(\zeta)$, so that there is an exact sequence

$$
0 \longrightarrow \Sigma^{-3} X \longrightarrow F \longrightarrow X \longrightarrow 0
$$

of DG-R-modules. It follows that $\mathrm{H}^{3}(F) \cong k \cong \mathrm{H}^{0}(F)$ and $\mathrm{H}^{i}(F)=0$ for $i \neq 0,3$. Now take $G$ to be a minimal DG- $R$-module associated with $F$. Evidently, $G$ has the same cohomology as $F$ and, since it is minimal, $\operatorname{rank}_{R} G=\operatorname{rank}_{k}\left(G \otimes_{R} k\right)$. The exact sequence (4.5) induces the exact sequence

$$
\begin{aligned}
\ldots \longrightarrow \mathrm{H}^{j+2}\left(\operatorname{End}_{R}(X)\right) & \stackrel{\lambda_{w}}{\longrightarrow} \mathrm{H}^{j}\left(\operatorname{End}_{R}(X)\right) \longrightarrow \mathrm{H}^{j}\left(\operatorname{Hom}_{R}(F, X)\right) \\
\longrightarrow & \mathrm{H}^{j+3}\left(\operatorname{End}_{R}(X)\right) \stackrel{\lambda_{w}}{\longrightarrow} \mathrm{H}^{j+1}\left(\operatorname{End}_{R}(X)\right) \longrightarrow \ldots
\end{aligned}
$$

Given this, Corollary 2.3 yields the inequality below

$$
\operatorname{rank}_{k}\left(G \otimes_{R} k\right)=\operatorname{rank}_{k} H\left(F \otimes_{R} k\right)=\operatorname{rank}_{k} H\left(\operatorname{Hom}_{R}(F, X)\right)<2^{d} .
$$

The first equality holds because the DG $R$-modules $F$ and $G$ are quasi-isomorphic and semifree; the second one is by adjunction, as $X$ and $k$ are quasi-isomorphic.

Remark 4.8. Halperin's toral rank conjecture [22, Problem 1.4] predicts that, for any topological space $X$ that is reasonable (say, a finite nilpotent CW complex) and that admits a free action of a $d$-dimensional torus $T$, the rational homology of $X$ satisfies

$$
\sum_{i} \operatorname{rank}_{\mathbb{Q}} H_{i}(X, \mathbb{Q}) \geqslant 2^{d}
$$


The validity of Conjecture 1.2 implies the toral rank conjecture: if $X$ admits such an action, then the relative minimal model of the corresponding Borel fibration is a semifree DG module $F$ over $R:=\mathbb{Q}\left[t_{1}, \ldots, t_{d}\right]$ with $\operatorname{rank}_{\mathbb{Q}} H(F)$ being finite and non-zero, and $\operatorname{rank}_{\mathbb{Q}} \mathrm{H}^{i}\left(\mathbb{Q} \otimes_{R} F\right)=\operatorname{rank}_{\mathbb{Q}} \mathrm{H}^{i}(X, \mathbb{Q})$; see $[3, \S 6]$ or $[19, \S 7.3 .2]$. Then, Conjecture 1.2 applied to a minimal DG $R$-module quasi-isomorphic to $F$ would yield the desired lower bound on $\sum_{i} \operatorname{rank}_{\mathbb{Q}} H_{i}(X, \mathbb{Q})$. However, our counterexamples do not affect the status of the toral rank conjecture, because the complex $G$ in Corollary 4.7 cannot be quasiisomorphic, even as a DG $R$-module, to any $F$ that arises as above.

Indeed, such an $F$ would come equipped with a morphism of DG algebras $\phi: R \rightarrow F$, and since $\mathrm{H}^{2}(F) \cong \mathrm{H}^{2}(G)=0$, by construction, a standard argument in the homotopy theory of DG algebras (see $[19, \S 2.2]$ ) implies that $\phi$ is homotopic to morphism that factors through $\mathbb{Q}$, and hence that

$$
\operatorname{rank}_{\mathbb{Q}} H\left(\mathbb{Q} \otimes_{R} F\right) \geqslant \operatorname{rank}_{\mathbb{Q}} \operatorname{Tor}^{R}(\mathbb{Q}, \mathbb{Q})=2^{d}
$$

This implies that $\operatorname{rank}_{R} G \geqslant 2^{d}$, contradicting the conclusion of Corollary 4.7.

Remark 4.9. In characteristic zero, Conjectures 1.2-1.5 admit families of counterexamples in which the value of the appropriate invariants deviate from the predicted one in an increasing fashion.

For example, for each $n \geqslant 1$, if $R$ is any regular local ring of dimension $2 n$ whose residue field $k$ has characteristic zero, then the construction in the proof of Theorem 4.1 gives a minimal finite free complex $G$ such that $H_{0}(G) \cong k \cong H_{1}(G)$ and $H_{i}(G)=0$ for all $i \neq 0,1$. Moreover, by Remark 2.5, one has

$$
\operatorname{rank}_{R}(G)=\left(\begin{array}{c}
2 n+2 \\
n+1
\end{array}\right)<2^{2 n} \frac{4}{\sqrt{\pi(n+1)}}
$$

The difference $2^{2 n}-\left(\begin{array}{c}2 n+2 \\ n+1\end{array}\right)$ tends to $\infty$ as $n$ goes to $\infty$, but $\left(\begin{array}{c}2 n+2 \\ n+1\end{array}\right)^{1 / 2 n}$ tends to 2 . This suggests the following question.

Is there a real number a>1 such that each finite free complex $F$ of modules over a regular local ring $R$ with $H(F)$ non-zero and of finite length satisfies

$$
\operatorname{rank}_{R}(F) \geqslant a^{\operatorname{dim} R} ?
$$

The family of examples constructed here shows that such an $a$ must satisfy

$$
a \leqslant \min _{n}\left\{\left(\begin{array}{c}
2 n+2 \\
n+1
\end{array}\right)^{1 / 2 n}\right\}<1.9605 .
$$


Remark 4.10. Let $R$ be a regular local ring of dimension $d \geqslant 8$ and $F$ the complex in Theorem 4.1. As $\mathfrak{m} H(F)=0$, one has that $H(F)$ is a module over $R / \mathfrak{m}$, and hence also over $R /(\boldsymbol{x})$, where $\boldsymbol{x}$ is any system of parameters for $R$. Since $R$ is regular, $\boldsymbol{x}$ is a regular sequence and the Koszul complex, say $E$, on $\boldsymbol{x}$ is a $R$-free resolution of $R /(\boldsymbol{x})$. However, there cannot be a DG $E$-module structure on $F$ : if there were, then $\operatorname{rank}_{R} F \geqslant 2^{d}$ by [11, Theorem 5.1], contrary to the conclusion of Theorem 4.1. See also [11, Remarks 5.3 and 5.4].

Acknowledgements. It is a pleasure to thank Lucho Avramov, Dave Benson and Seth Lindokken for helpful conversations, which extended the scope of this work. We are grateful to Volker Puppe and Matthias Franz for comments and suggestions. We are also indebted to one of the referees who gave us extensive feedback on the first version of this manuscript, leading to substantial revisions. S. B. I. was partially supported by NSF grant DMS-1700985 and M. E. W. was partially supported by grant \#318705 from the Simons Foundation.

\section{References}

[1] Adem, A. \& Browder, W., The free rank of symmetry of $\left(S^{n}\right)^{k}$. Invent. Math., 92 (1988), 431-440.

[2] Adem, A. \& Swan, R. G., Linear maps over abelian group algebras. J. Pure Appl. Algebra, 104 (1995), 1-7.

[3] Allday, C. \& Halperin, S., Lie group actions on spaces of finite rank. Quart. J. Math. Oxford Ser., 29 (1978), 63-76.

[4] Allday, C. \& Puppe, V., On the localization theorem at the cochain level and free torus actions, in Algebraic Topology (Göttingen, 1984), Lecture Notes in Math., 1172, pp. 1-16. Springer, Berlin-Heidelberg, 1985.

[5] - Bounds on the torus rank, in Transformation Groups (Poznań, 1985), Lecture Notes in Math., 1217, pp. 1-10. Springer, Berlin-Heidelberg, 1986.

[6] Avramov, L. L., Modules of finite virtual projective dimension. Invent. Math., 96 (1989), $71-101$.

[7] - Local rings over which all modules have rational Poincaré series. J. Pure Appl. Algebra, 91 (1994), 29-48.

[8] - Infinite free resolutions, in Six Lectures on Commutative Algebra (Bellaterra, 1996), Progr. Math., 166, pp. 1-118. Birkhäuser, Basel, 1998.

[9] Avramov, L. L. \& Buchweitz, R. O., Homological algebra modulo a regular sequence with special attention to codimension two. J. Algebra, 230 (2000), 24-67.

[10] Avramov, L. L., Buchweitz, R. O. \& Iyengar, S., Class and rank of differential modules. Invent. Math., 169 (2007), 1-35.

[11] Avramov, L. L., Iyengar, S. B. \& Neeman, A., Big Cohen-Macaulay modules, morphisms of perfect complexes, and intersection theorems in local algebra. Preprint, 2017. arXiv: 1711.04052 [math.AC]

[12] BollobÁs, B., Random Graphs. Cambridge Studies in Advanced Mathematics, 73. Cambridge Univ. Press, Cambridge, 2001. 
[13] Bruns, W. \& Herzog, J., Cohen-Macaulay Rings. Cambridge Studies in Advanced Mathematics, 39. Cambridge Univ. Press, Cambridge, 1993.

[14] Buchsbaum, D. A. \& Eisenbud, D., Algebra structures for finite free resolutions, and some structure theorems for ideals of codimension 3. Amer. J. Math., 99 (1977), $447-$ 485.

[15] Carlsson, G., Free $(\mathbf{Z} / 2)^{k}$-actions and a problem in commutative algebra, in Transformation Groups (Poznań 1985), Lecture Notes in Math., 1217, pp. 79-83. Springer, Berlin-Hidelberg, 1986.

[16] - Free $(\mathbf{Z} / 2)^{3}$-actions on finite complexes, in Algebraic Topology and Algebraic K-Theory (Princeton, NJ, 1983), Ann. of Math. Stud., 113, pp. 332-344. Princeton Univ. Press, Princeton, NJ, 1987.

[17] Conca, A., Herbig, H.-C. \& Iyengar, S. B., Koszul properties of the moment map of some classical representations. Collect. Math., 69 (2018), 337-357.

[18] Evans, E. G. \& Griffith, P., Syzygies. London Math. Soc. Lecture Note Ser., 106. Cambridge Univ. Press, Cambridge, 1985.

[19] FÉlix, Y., Oprea, J. \& Tanré, D., Algebraic Models in Geometry. Oxford Graduate Texts in Mathematics, 17. Oxford Univ. Press, Oxford, 2008.

[20] Griffiths, P. \& Harris, J., Principles of Algebraic Geometry. Wiley Classics Library. John Wiley \& Sons, New York, 1994.

[21] Gulliksen, T.H., A change of ring theorem with applications to Poincaré series and intersection multiplicity. Math. Scand., 34 (1974), 167-183.

[22] Halperin, S., Rational homotopy and torus actions, in Aspects of Topology, London Math. Soc. Lecture Note Ser., 93, pp. 293-306. Cambridge Univ. Press, Cambridge, 1985.

[23] Hartshorne, R., Algebraic vector bundles on projective spaces: a problem list. Topology, 18 (1979), 117-128.

[24] Roberts, P., Homological Invariants of Modules over Commutative Rings. Séminaire de Mathématiques Supérieures, 72. Presses de l'Université de Montréal, Montreal, QC, 1980.

[25] Serre, J. P., Complex Semisimple Lie Algebras. Springer, New York, 1987.

[26] Walker, M. E., Total Betti numbers of modules of finite projective dimension. Ann. of Math., 186 (2017), 641-646.

[27] — Total Betti numbers of modules of finite projective dimension. Preprint, 2017. arXiv: 1702.02560 [math. AC].

SRIKANTh B. IYengaR

Department of Mathematics

University of Utah

Salt Lake City, UT 84112

U.S.A.

iyengar@math.utah.edu

Received June 7, 2017

Received in revised form February 17, 2018
MARK E. WALKER

Department of Mathematics

University of Nebraska

Lincoln, NE 68588

U.S.A.

mark. walker@unl .edu 